\title{
ARTICLE
}

\section{At-risk mental state for psychosis: identification and current treatment approaches}

\author{
Andrew Thompson, Steven Marwaha \& Matthew R. Broome
}

Andrew Thompson is an Associate Clinical Professor in Psychiatry at the University of Warwick and a consultant psychiatrist in the North Warwickshire early intervention in psychosis service. He previously worked at the Personal Assessment and Crisis Evaluation (PACE) clinic in Melbourne, a research clinic for patients at risk for psychosis. His research interests include risk factors and biomarkers for developing psychosis. Steven

Marwaha is an Associate Clinical Professor at the University of Warwick and a consultant psychiatrist in Coventry. His research interests are in early interventions

for bipolar disorders and identifying risk states for bipolar disorder.

Matthew R. Broome is a Senior

Clinical Research Fellow at the

University of Oxford and an Honorary Associate Clinical Professor of

Psychiatry at the University of

Warwick. He works as a consultant psychiatrist in the Oxford early

intervention in psychosis service. He previously worked at the OASIS at-risk service in London and has an interest in the ethics of at-risk

states and biomarkers for predicting psychosis.

Correspondence Dr Andrew Thompson, Division of Mental Health and Wellbeing, Warwick Medical School, Gibbet Hill, Coventry CV47AL, UK. E-mail andrew.d.thompson@warwick.ac.uk

\section{SUMMARY}

The concept of an 'at-risk mental state' for psychosis arose from previous work attempting to identify a putative psychosis prodrome. In this article we summarise the current criteria used to identify 'at-risk' individuals, such as the ultra-highrisk (UHR) criteria, and the further identification of important clinical risk factors or biomarkers to improve prediction of who might develop a psychotic disorder. We also discuss important ethical issues in classifying and treating at-risk individuals, current treatment trials in this area and what treatment current services can offer.

\section{LEARNING OBJECTIVES}

- Understand the development, refinement and use of tools attempting to identify a putative psychosis prodrome or 'at-risk mental state'

- Appreciate the ethical issues in identifying and treating individuals with an 'at-risk mental state' for psychosis

- Consider the treatment options in light of the ethical issues, the research trials and what current services can offer

\section{DECLARATION OF INTEREST}

None

It has long been known that the majority of psychotic disorders do not develop de novo. There is invariably a period of non-specific or low-grade symptoms, or 'prodrome', prior to the onset of a frank psychosis. In medicine, a prodrome is an early symptom (or set of symptoms) that might indicate the start of a disease before specific symptoms occur. A common example would be measles, which is described as having a prodrome of 3-4 days consisting of non-specific symptoms such as fever, coryzal symptoms, conjunctivitis and cough. This is followed by the specific rash, making the definitive diagnosis possible.

Attempts to identify a prodrome in psychosis are not new. The notion of being able to prevent the onset of schizophrenia and other psychotic disorders by detecting and intervening in the prodromal phase has been a goal discussed for many years (Sullivan 1927). Half a century ago Chapman and others outlined the developing symptoms of schizophrenia (Chapman 1966; Yung 1996) retrospectively. They are often non-specific symptoms such as depression, anxiety and disturbance in sleep patterns, as well as psychotic symptoms that are fleeting or not fully formed. In this article we will summarise the work to date on attempts to prospectively identify the prodrome, concentrating on the so-called 'at-risk mental state' group, including the effectiveness of interventions. This is especially important to UK psychiatrists at present as the new mental health targets for psychosis include this group, so services for these patients may become more widespread (Marwaha 2016).

\section{Identification of a putative prodrome}

In the mid-1990s researchers attempted to characterise or identify a 'putative prodrome' for psychosis prospectively. This was prompted by a critique of the DSM-III prodrome category, the emerging 'early intervention in psychosis' paradigm and further retrospective accounts of prodromal symptoms in patients with first-episode psychosis. This led to the idea that identifying an 'at-risk mental state' for psychosis, based on some of the reported prodromal symptoms, might be useful in order to predict subsequent development of a psychotic disorder. The goal was to identify a group at imminent high risk of developing a psychotic disorder, using a combination of genetic and clinical risk factors (Yung 2003). Criteria have since been developed to attempt to identify this group, such as the ultrahigh-risk (UHR) criteria (Yung 2004a) or the similar clinical high-risk (CHR) criteria (Miller 2003); those meeting these criteria are deemed to have an at-risk mental state (ARMS) for psychosis. In this article we will use the UHR criteria to identify this group, as these were the first criteria to be developed (at the Personal Assessment and Crisis Evaluation (PACE) clinic in Melbourne, Australia) and they are most commonly used in the UK. The criteria were named 'ultra-high-risk' to distinguish 
this help-seeking clinical group from other highrisk populations such as those with a genetic risk.

In brief, the UHR criteria (Box 1) comprise three groups:

- attenuated psychotic symptoms (APS): the presence of attenuated (subthreshold for a diagnosis of a psychotic disorder) psychotic symptoms within the previous 12 months

- brief limited intermittent psychotic symptoms (BLIPS): a history of brief self-limiting psychotic symptoms in the previous 12 months, which resolve spontaneously (within 7 days)

- trait group: genetic vulnerability to psychotic disorder (either schizotypal personality disorder or family history of psychotic disorder in a firstdegree relative).

\section{BOX 1 The Melbourne ultra-high-risk (UHR) criteria}

The individual must:

- be aged between 15 and 25 years

- have been referred to a specialised service for help

- have experienced a drop in functioning of at least 1 month over the past year or sustained low functioning - meet the criteria for one or more of groups 1-3 below

Group 1: Attenuated psychotic symptoms (APS)

- Presence of at least one of the following symptoms ideas of reference, odd beliefs or magical thinking, perceptual disturbance, paranoid ideation, odd thinking and speech, odd behaviour and appearance

- Frequency of symptoms: at least several times a week

- Recency of symptoms: present within the past year

- Duration of symptoms: present for at least 1 week and no longer than 5 years

Group 2: Brief limited intermittent psychotic symptoms (BLIPS)

- Transient psychotic symptoms

- Presence of at least one of the following: ideas of reference, magical thinking, perceptual disturbance, paranoid ideation, odd thinking or speech

- Duration of episode: less than 1 week

- Frequency of symptoms: at least several times a week

- Symptoms resolve spontaneously

- Recency of symptoms: must have occurred within the past year

Group 3: Trait vulnerability group

- Schizotypal personality disorder in the identified individual, or a first-degree relative with a psychotic disorder

(Adapted from Nelson et al (2012))
A drop in functioning or persistent low functioning for at least 1 month within the previous 12 months is required in all three groups. Age is also a criterion, as this focuses on those in the highest epidemiological risk period for development of psychosis. At the PACE clinic in Melbourne, the age range is currently 15-25 and most of the research cited below focuses on this young population.

The initial studies using these criteria suggested that they identified a group at high risk of transition to (development of) a full-threshold psychotic disorder, defined as full psychotic symptoms occurring for over 1 week, which was taken as the threshold for when clinicians would generally start treatment. A recent meta-analysis of transition to a psychotic disorder from studies in this population, using a variety of similar tools for measuring the at-risk mental state, reported transition rates of $18 \%$ after 6 months of follow-up, 22\% after 1 year, $29 \%$ after 2 years and 36\% after 3 years (FusarPoli 2012). These risks are around 400 times the population risk for development of psychosis. The risks also appear to continue over a longer period (Nelson 2013).

Rating scales have been developed and validated to assess and identify those who meet the UHR criteria. The most widely used is the Comprehensive Assessment of At-Risk Mental States (CAARMS) (Yung 2005), developed in Melbourne, although the similar Structured Interview for Prodromal Symptoms (SIPS) and the associated Scale of Prodromal Symptoms (SOPS) (Miller 2003) are more often used in the USA and some other international centres. These scales focus on the positive symptoms of psychosis (disordered beliefs, perceptions and speech) as the way of clinically indexing risk.

\section{The basic symptoms and self-disturbance approach}

A different approach to understanding and characterising the prodrome has been proposed by European research groups. This highlights the importance of subtle subjective alterations in cognition, memory and thinking that may be manifest many years before the onset of a psychotic disorder (Huber 1989). These have been termed 'basic symptoms'. Research groups have developed assessment tools to identify those presenting with such symptoms, for example the Schizophrenia Proneness Instrument - Adult version (SPI-A) (Schultze-Lutter 2007). Individuals meeting basic symptoms criteria have a much higher risk of developing a psychotic disorder in the future: one study reported that the presence of at least 1 of 66 basic symptoms at baseline predicted the development of schizophrenia at 9.6 year 
follow-up with a specificity of $59 \%$, a sensitivity of $98 \%$, a positive predictive value (PPV) of $70 \%$ and a negative predictive value (NPV) of $96 \%$ (Klosterkotter 2001). Some research groups have combined the two concepts and classified those who meet these basic symptoms criteria as being in an early initial prodromal stage, with the UHR criteria identifying a late initial prodromal stage (Bechdolf 2012).

A related set of criteria examine the disturbance of the sense of self in putatively prodromal patients using tools such as the Examination of Anomalous Self-Experience (EASE) (Parnas 2005). We will concentrate on the UHR/ARMS concept here as it is more widely used in UK practice, although the approach does relatively neglect those presenting with cognitive problems or negative symptoms.

At present, applying the assessment and criteria for identification of basic symptoms and 'selfdisturbances' requires considerable training and they are therefore less accessible to clinicians in practice, but their use remains of considerable interest in some countries.

\section{Problems with the UHR criteria and improving predictive power}

Despite the initial enthusiasm for prospectively identifying UHR individuals there were criticisms, especially when intervention and treatment strategies were proposed for them. First, although transition rates were high, it was still the case that more than half of the identified individuals did not develop psychosis within 12 months. This may not be a problem for some disorders but, for a potentially stigmatising disorder such as psychosis, especially if treatment is considered, it is important. Second, there were reports that the rate of transition to psychosis may not be as high as first thought. This appears to be a particular problem in established clinics, where transition rates have declined over time (Yung 2007). There are a number of explanations for this, but one important factor is the population from which the sample is drawn. Similar to any diagnostic test, the positive predictive power of these criteria is affected by the population prevalence of the disorder. Therefore, if these criteria were applied to a non-help-seeking population sample then they would (and do) have a lower predictive power than if they were used in a sample referred to an early psychosis clinic but not quite meeting the criteria for a psychotic disorder. This has been discussed in the literature (Yung 2007). In response to this there have been attempts to see whether the predictive power of these criteria can be improved by using clinical factors and/or biomarkers.
A study of 104 UHR individuals reported by the group in Melbourne investigated whether particular clinical or demographic factors, in addition to the UHR criteria, could be used to improve the prediction of which of these individuals would develop a psychotic disorder (Yung 2004b). Four baseline clinical predictors of transition to psychosis were identified: a combination of attenuated psychotic symptoms and genetic risk; a long duration of symptoms prior to baseline; poor social functioning; and poor attention. A model requiring the presence of at least one of these four potential predictors gave a good predictive validity, with a PPV of $80.8 \%$ and a sensitivity and specificity of $60.0 \%$ and $92.6 \%$ respectively. Belonging to at least one of the three inclusion groups and belonging to the BLIPS group also increased the risk of transition.

The North American Prodrome Longitudinal Study (NAPLS) consortium investigated the predictive power of a large number of variables using their pooled sample of 291 cases (Cannon 2008). This was a particularly important study given that one of the methodological difficulties in UHR research to that point had been small sample sizes. The consortium found that five variables were strong predictors of transition to psychosis and that when these variables were combined the PPV was as high as $81 \%$, without a substantial compromise in sensitivity or specificity. These predictors had substantial, but not complete, overlap with the predictors found from the earlier Melbourne study described above (Yung 2004b). Three of the five variables were found to be associated with transition in a replication study: high unusual thought content scores on the rating scales; low functioning; and having genetic risk with functional decline (Thompson 2011).

The European Prediction of Psychosis Study (EPOS) group, again using multisite data, investigated predictors in a European sample. They reported a high PPV (83.3\%) for a six-variable model that included positive symptoms, bizarre thinking, sleep disturbances, schizotypal personality disorder, Global Assessment of Functioning (GAF) score and years of education. They also reported a different and innovative method of assessing 'risk' using a prognostic index, which enables the risk of individual patients to be calculated (Ruhrmann 2010). The addition of UHR criteria and basic symptoms criteria, as well as the related subjective symptoms of self-disturbance, has also been shown to increase predictive power regarding transition to psychosis (Nelson 2012).

Interestingly, in another study from the PACE clinic in Melbourne, the initial judgements of 
experienced clinicians as to whether an individual meeting UHR criteria and admitted to the clinic would subsequently develop psychosis were adequate (a sensitivity of $80 \%$, specificity of $84 \%$, PPV of 32\%) but not extremely accurate predictors, so caution about the accuracy of clinical prediction based on 'praecox feeling' was recommended (Nelson 2010).

In summary, it appears that additional specific clinical factors such as specific positive psychotic symptoms, poor functioning, negative symptoms and subtle disturbances in cognition or sense of self may improve the predictive value of the UHR criteria. Further categorising these individuals using individual risk profiles is an area of research development.

\section{Risk factors or biomarkers}

A number of biomarkers or phenotypic markers have been investigated to see whether they can increase the predictive ability of UHR criteria. Biomarkers of note include structural imaging changes such as parahippocampal grey matter volume (Mechelli 2011), with studies synthesising data from multiple sites confirming the role that neuroanatomical changes may have in the prediction of psychosis (e.g. Koutsouleris 2015). Functional neuroimaging has also been utilised to predict psychosis - both in measuring in vivo neurochemistry and in task and resting-state functional magnetic resonance imaging (fMRI). Functional imaging changes that may predict psychosis include changes in prefrontal and cortical function on the verbal fluency task and their relation to dopamine levels (Fusar-Poli 2011; Allen 2012), improvement in left inferior frontal gyrus function correlated with reduction in prodromal symptoms longitudinally (Fusar-Poli 2011), and, on functional analysis of networks, a change in the centrality of the anterior cingulate cortex in the network (Lord 2012). Using positron emission tomography (PET) and magnetic resonance spectroscopy (MRS) to study neurochemical changes, reduction in presynaptic dopamine levels predicted transition to psychosis (Howes 2011) and lower levels of thalamic glutamate were associated with a poorer functional outcome (Allen 2015). Electrophysiological markers such as p300, sensory gating and mismatch negativity (MMN) have all been shown to be impaired in those at risk for psychosis, with particularly strong evidence that MMN can predict onset of disorder (Bodatsch 2015). Other biomarkers include inflammatory and oxidative stress markers and genetic variants such as the neuregulin gene (Keri 2009). A recent study found that 15 markers, including inflammation, oxidative stress, hormones and metabolic analytes, potentially served as a blood assay to predict psychosis (Perkins 2015). Phenotypic markers include social cognitive and neurocognitive deficits such as poor theory of mind, working memory or executive functioning and verbal fluency (Giuliano 2012).

None of the biomarkers mentioned here is at present used practically in routine clinic settings, although research is ongoing to attempt to combine some of these markers with clinical factors to be of use to clinicians.

\section{Intervention studies}

There have now been a number of randomised clinical trials of interventions in the UHR group, ranging from antipsychotics to cognitivebehavioural therapy (CBT) and omega-3 fatty acids, with the main aim to prevent or delay the onset of psychosis. These are shown in online Table DS1. For pharmacological interventions we have restricted the trials included in the table to placebocontrolled designs.

There are currently three meta-analyses comparing these treatments using the outcome of transition to a full-threshold psychotic disorder (Preti 2010; Stafford 2013; van der Gaag 2013), and these suggest that there is some promise for all current interventions; the meta-analysis of Van der Gaag and colleagues reported a number needed to treat (NNT) of 9 to prevent psychosis at 12 months. Early enthusiasm for the use of lowdose antipsychotics was tempered by the non-trivial rates of side-effects (weight gain with olanzapine and extra-pyramidal symptoms with risperidone) (McGorry 2002; McGlashan 2006) and the finding that the short-term reduction in the transition rate was not maintained over time (Phillips 2007). Similarly, initial enthusiasm in small trials of CBT (Morrison 2004) have been followed by less striking results (at least in terms of transition rates) in larger trials (Morrison 2012), although other trials have found more positive results on transition rates (van der Gaag 2012). A single placebo-controlled trial of omega-3 fatty acids (Amminger 2010) was particularly promising, but initial results from the first of two replication studies failed to find a significant positive effect in terms of transition to psychosis at 12 months (McGorry 2015).

Non-randomised trials without placebo controls have suggested the benefits of antidepressant medications (Cornblatt 2007) and amisulpride (Ruhrmann 2007). The efficacy of other pharmacological interventions, such as glycine, and of nonpharmacological approaches such as family therapy is currently being investigated. One problem with all trials that specifically target a diminished 
transition rate is whether the intervention results in a transition time shifted to after the trial ends or whether the protection rendered is long term.

The decreased rate of transition to psychotic disorder in recent years (Yung 2007) has meant that many more intervention trials in the UHR group are possibly underpowered. In a number of recent trials the standard befriending, supportive therapy, active monitoring or case-management strategies have fared as well as the intervention strategy when the outcome has been transition to a psychotic disorder. This has prompted some involved in these trials to suggest an alternative to the opinion that their studies are underpowered; instead, they hypothesise that the relatively non-specific 'control' interventions such as active monitoring and supportive therapy (which are not in fact 'treatment as usual' but low-grade interventions) may be effective in some individuals who meet the UHR criteria (Morrison 2012; McGorry 2013).

\section{Ethical issues}

It is worth highlighting again that the idea of treating psychotic symptoms very early in the prodrome and in UHR patients has been the subject of much discussion from both clinical and ethical perspectives (Yung 2007). Some feel that the approach advocates treating people too early and labelling and/or potentially stigmatising individuals (Yang 2013) when less than 50\% will develop a psychotic disorder in the short to medium term. These are the so-called 'false-positive' at-risk individuals - i.e. those who are not prodromal for eventual psychosis and for whom there is therefore the risk of over-treatment. Also, where antipsychotics are used as an intervention, there is a risk of iatrogenic dopamine sensitisation, symptom rebound on drug withdrawal and brain changes on exposure to antipsychotics (Moncrieff 2006). More recently, attention has turned to the persistence and development of other psychiatric disorders in this group and the poor functional outcome of these patients regardless of whether they develop psychosis (Lin 2015). The percentage of patients meeting UHR criteria who meet the criteria for another mental disorder is high. This reinforces the idea that these individuals may be at risk for psychosis, but are certainly at risk for other poor outcomes (Lin 2015). This has led to discussion about the need to target functioning as well as a defined psychosis threshold in this population.

There was much debate among all stakeholders during the preparation for the DSM-5 diagnostic manual (American Psychiatric Association (APA) 2013) as to whether a 'risk syndrome for psychosis' should be included in the main body of the manual.
The debate centred on whether it was premature to include this as a disorder solely on the basis of the predictive validity of the criteria (Yung 2010). A version of the UHR criteria describing 'attenuated psychosis syndrome' was eventually included, but in section III (the conditions-for-further-study section of DSM-5), indicating that further study is needed before possible inclusion in the main document. Some have argued that the DSM-5 diagnosis of 'other specified schizophrenia spectrum disorder and other psychotic disorders' (298.8 in DSM-5, F28 in ICD-10) includes 'attenuated psychosis syndrome'. The APA criteria will be subject to further field trials before the next revision of DSM.

A further ethical concern that many clinicians working in this area routinely face is the tension between reassurance and normalisation of the unusual experiences on the one hand, and the followup, monitoring and genuine appreciation of risk necessary in high-risk patients on the other. If not delivered with skill and expertise, the monitoring (and the care offered) may itself have an impact on anxiety, psychological appraisals and so on, and thus may have the paradoxical effect of increasing, rather than decreasing, the rate of transition. Expanding clinical awareness and service delivery may lead to this and other unwanted, and unforeseen, outcomes, one of which may be how the criminal justice system decides to treat an offender who demonstrates 'attenuated psychosis syndrome': will it be viewed as a mental disorder, with all the attendant consequences to the patient, the courts and the clinicians? Or will the legal system see it as the risk state that it is? More subtle ethical issues surround how one talks about risk with young people deemed to be at risk. As noted above, the majority of such individuals do not develop a frank psychosis. Hence, should clinicians who work in early detection consider whether patients have a right not to know their prognosis? One could argue that, since no specific treatments have clearly been shown to alter clinical course, there may be no positive benefit in knowing. Further, there are the possible harms of self-stigmatisation and fear from the potential diagnosis. Despite these points, many would argue that, if sensitive and appropriate interventions can delay or reduce the impact of developing psychosis, even in a minority of individuals, this is warranted given the significance for most individuals of a psychotic disorder.

\section{Current specialised UHR services}

Although the concepts of UHR and ARMS have been more widely accepted, there are few services that provide specific interventions for identified patients in the UK. Provision is mostly aligned 
to early intervention in psychosis (EIP) services (increasingly called simply early intervention services). OASIS Outreach and Support in south London, Early Detection and Intervention Team (EDIT) services in Birmingham and Manchester and the CAMEO early intervention service in Cambridge are notable exceptions of specific UHR teams. These services often have a strong research focus. Worldwide there has been considerable expansion of such services and this trend seems likely to continue. Specialised clinics offer CBTfocused interventions along with case management and treatment of psychiatric comorbidities. An example of an approach from the specialised PACE clinic in Melbourne is shown in Box 2. These specialised clinics have been shown to be costeffective (Valmaggia 2009), which has added to the argument for providing specific services for UHR patients. Assessment and treatment of such patients has been included as part of the new Department of Health waiting-time standards for first-episode psychosis (Department of Health 2014).

\section{What should a clinician do where no specialised services are available?}

What are the options then for clinicians who see UHR patients in their clinic and do not have the option of referring them to a specialised service? Often the local early intervention team will have a policy on what treatment to suggest and may provide an assessment using the CAARMS or a similar tool. However, often this is a 'watch and wait' approach and the level of specific intervention is minimal. With the advent of the new waiting target for first-episode psychosis it is likely that more services will be proving specific interventions for people who meet the UHR criteria (Department of Health 2014).

The matter of risk needs to be handled sensitively, as highlighted above. For psychiatrists, the temptation might be to start someone with subthreshold symptoms on a low dose of an antipsychotic, especially when other non-pharmacological interventions are not available. The current research suggests that: (a) more than half of UHR patients will not develop a psychotic illness even in the long term and therefore psychiatrists may be treating a high proportion inappropriately and not in fact delaying or reducing transition to psychosis; and (b) although there are some indicators of those at highest risk, current tools and clinical predictors are less than perfect. On balance, the suggestion is that antipsychotics should not be used in the first instance. Current international guidelines also suggest that antipsychotics should not be first line, but if rapid worsening of psychotic symptoms
B0X 2 The PACE clinic's approach to the treatment of people at ultra-high risk of psychosis
1 Assessment, formulation and engagement

2 Psychoeducation

3 Individual case management - includes:

- ongoing monitoring of the client's mental state and risks

- ensuring the client and family or carers are appropriately informed about the nature of the mental health problems and their treatment

- reducing the trauma or anxiety associated with any necessary in-patient admissions

- facilitating adequate treatment for comorbid disorders

- assisting in reducing any adverse impact of the illness on the person's psychosocial environment, for example in relationships, accommodation, education, employment, financial security

- fostering the person's recovery, reintegration into society and restoration of a normal developmental trajectory

- risk assessment and management

4 Family interventions

5 CBT using a stress-vulnerability mode

- stress management

- positive symptoms

- depression/negative symptoms

- basic symptoms

- comorbidities

(Adapted from PACE Manual Writing Group 2012) occurs together with significant deterioration in functioning related to these symptoms and elevated risk to self or others, a low-dose atypical antipsychotic may be considered, in conjunction with close monitoring and support' (Early Psychosis Guidelines Writing Group 2010: p. 45). This is also supported by the National Institute for Health and Care Excellence (NICE), whose clinical guideline on psychosis and schizophrenia in adults states: 'Do not offer antipsychotic medication to: people considered to be at increased risk of developing psychosis [...] or with the aim of decreasing the risk of or preventing psychosis' (NICE 2014: p. 15).

Treating the common comorbidities such as depression and anxiety, if at treatment thresholds, is definitely warranted and a more watchful waiting approach is advised, as diagnostic uncertainty is common.

As mentioned earlier, high-dose omega-3 fatty acids initially seemed promising in preventing transition to psychosis and they were relatively side-effect free, but recent research does not appear to have strongly replicated the initial positive findings. In services where specialised psychological interventions are available, CBT would be appropriate. With UHR individuals, cognitive-behavioural approaches often focus on other difficulties such as depression and anxiety rather than simply psychotic symptoms (Box 2).

For some patients, often those whose symptoms are exacerbated by stress or drug use, more practical solutions offered in the process of assessment or in care coordination, or interventions for substance misuse can have beneficial effects. 


\section{MCQ answers}

$1 \mathrm{c} \quad 2 \mathrm{c} \quad 3$ e 4 a 5 e
The NICE guideline now includes the following recommendations on treatment of at-risk patients: 'offer individual CBT with or without family intervention $[. .$.$] and offer interventions recommended$ in NICE guidance for people with any of the anxiety disorders, depression, emerging personality disorder or substance misuse' (NICE 2014: p. 15).

\section{Conclusions}

Attempts to prospectively identify individuals at very high risk of developing a psychotic disorder have considerably advanced the research knowledge on both the mechanism of development of psychosis and approaches to very early or indicated intervention. Although there was initial enthusiasm with regard to the ability to identify and treat clinical presentations such as the UHR state, some caution has been raised regarding the predictive validity of the current criteria and the consequences of using such labels. Further refining of the criteria, including the use of biomarkers, phenotypes and clinical features, is the next step in the pathway towards the overall goal of altering the course of psychotic disorders.

\section{References}

Allen P, Luigjes J, Howes OD, et al (2012) Transition to psychosis associated with prefrontal and subcortical dysfunction in ultra high-risk individuals. Schizophrenia Bulletin, 38: 1268-76.

Allen P, Chaddock CA, Egerton A, et al (2015) Functional outcome in people at high risk for psychosis predicted by thalamic glutamate levels and prefronto-striatal activation. Schizophrenia Bulletin, 41: 429-39.

American Psychiatric Association (2013) Diagnostic and Statistical Manual of Mental Disorders (5th edn) (DSM-5). APA.

Amminger GP, Schafer MR, Papageorgiou K, et al (2010) Long-chain \{omega\}-3 fatty acids for indicated prevention of psychotic disorders: a randomized, placebo-controlled trial. Archives of General Psychiatry, 67: 146-54.

Bechdolf A, Wagner M, Ruhrmann S, et al (2012) Preventing progression to first-episode psychosis in early initial prodromal states. British Journal of Psychiatry 200: 22-9.

Bodatsch M, Brockhaus-Dumke A, Klosterkotter J, et al (2015) Forecasting psychosis by event-related potentials: systematic review and specific meta-analysis. Biological Psychiatry, 77: 951-8.

Cannon TD, Cadenhead K, Cornblatt B, et al (2008) Prediction of psychosis in youth at high clinical risk: a multisite longitudinal study in North America. Archives of General Psychiatry, 65: 28-37.

Chapman J (1966) The early symptoms of schizophrenia. British Journal of Psychiatry, 112: 225-51.

Cornblatt BA, Lencz T, Smith CW, et al (2007) Can antidepressants be used to treat the schizophrenia prodrome? Results of a prospective, naturalistic treatment study of adolescents. Journal of Clinical Psychiatry, 68: 546-57.

Department of Health (2014) Achieving Better Access to Mental Health Services by 2020. Department of Health.

Early Psychosis Guidelines Writing Group (2010) Australian Clinical Guidelines for Early Psychosis (2nd edn). Orygen Youth Health.

Fusar-Poli P, Broome MR, Matthiasson P, et al (2011) Prefrontal function at presentation directly related to clinical outcome in people at ultra high risk of psychosis. Schizophrenia Bulletin, 37: 189-98.
Fusar-Poli P, Bonoldi I, Yung AR, et al (2012) Predicting psychosis: meta-analysis of transition outcomes in individuals at high clinical risk. Archives of General Psychiatry, 69: 220-9.

Giuliano AJ, Li H, Mesholam-Gately RI, et al (2012) Neurocognition in the psychosis risk syndrome: a quantitative and qualitative review. Current Pharmaceutical Design, 18: 399-415

Howes 0, Bose S, Turkheimer F, et al (2011) Progressive increase in striatal dopamine synthesis capacity as patients develop psychosis: a PET study. Molecular Psychiatry, 16: 885-6.

Huber G, Gross G (1989) The concept of basic symptoms in schizophrenic and schizoaffective psychoses. Recenti Progressi in Medicina, 80: 646-52.

Keri S, Kiss I, Kelemen 0 (2009) Effects of a neuregulin 1 variant on conversion to schizophrenia and schizophreniform disorder in people at high risk for psychosis. Molecular Psychiatry, 14: 118-9.

Klosterkotter J, Hellmich M, Steinmeyer EM, et al (2001) Diagnosing schizophrenia in the initial prodromal phase. Archives of General Psychiatry, 58: 158-64

Koutsouleris N, Riecher-Rossler A, Meisenzahl EM, et al (2015) Detecting the psychosis prodrome across high-risk populations using neuroanatomical biomarkers. Schizophrenia Bulletin, 41: 471-82.

Lin A, Wood SJ, Nelson B, et al (2015) Outcomes of nontransitioned cases in a sample at ultra-high risk for psychosis. American Journal of Psychiatry, 172: 249-58.

Lord LD, Allen P, Expert P, et al (2012) Functional brain networks before the onset of psychosis: a prospective $\mathrm{fMRI}$ study with graph theoretical analysis. Neuroimage Clinical, 1: 91-8.

Marwaha S, Thompson A, Upthegrove R, et al (2016) 15 years on - early intervention for a new generation. British Journal of Psychiatry, in press.

McGlashan TH, Zipursky RB, Perkins D, et al (2006) Randomized, double-blind trial of olanzapine versus placebo in patients prodromally symptomatic for psychosis. American Journal of Psychiatry, 163: 790-9.

McGorry PD, Yung AR, Phillips LJ, et al (2002) Randomized controlled trial of interventions designed to reduce the risk of progression to firstepisode psychosis in a clinical sample with subthreshold symptoms. Archives of General Psychiatry, 59: 921-8.

McGorry PD, Nelson B, Phillips LJ, et al (2013) Randomized controlled trial of interventions for young people at ultra-high risk of psychosis: twelve-month outcome. Journal of Clinical Psychiatry, 74: 349-56.

McGorry P, Markulev C, Nelson B, et al (2015) NEURAPRO-E study protocol: a multicentre randomized controlled trial of omega-3 fatty acids and cognitive-behavioural case management for patients at ultra high risk of schizophrenia and other psychotic disorders. Schizophrenia Bulletin, 41 (suppl 1): S322-3.

Mechelli A, Riecher-Rossler A, Meisenzahl EM, et al (2011) Neuroanatomical abnormalities that predate the onset of psychosis: A multicenter study. Archives of General Psychiatry, 68: 489-95.

Miller TJ, McGlashan TH, Rosen JL, et al (2003) Prodromal assessment with the structured interview for prodromal syndromes and the scale of prodromal symptoms: predictive validity, interrater reliability, and training to reliability. Schizophrenia Bulletin, 29: 703-15.

Moncrieff J (2006) Does antipsychotic withdrawal provoke psychosis? Review of the literature on rapid onset psychosis (supersensitivity psychosis) and withdrawal-related relapse. Acta Psychiatrica Scandinavica, 114: 3-13.

Morrison AP, French P, Walford L, et al (2004) Cognitive therapy for the prevention of psychosis in people at ultra-high risk: randomised controlled trial. British Journal of Psychiatry, 185: 291-7.

Morrison AP, French P, Stewart SLK, et al (2012) Early detection and intervention evaluation for people at risk of psychosis: multisite randomised controlled trial. BMJ, 344: e2233.

National Institute for Health and Care Excellence (2014) Psychosis and Schizophrenia in Adults: Treatment and Management (NICE Clinical Guideline CG178). NICE.

Nelson B, Yung AR (2010) Can clinicians predict psychosis in an ultra high risk group? Australian and New Zealand Journal of Psychiatry, 44: 625-30 
Nelson B, Thompson A, Yung AR (2012) Basic self-disturbance predicts psychosis onset in the ultra high risk for psychosis 'prodromal' population. Schizophrenia Bulletin, 38: 1277-87.

Nelson B, Yuen HP, Wood SJ, et al (2013) Predictors of transition to psychosis in the ultra high risk ("prodromal") population: a long term follow up study. Archives of General Psychiatry, 70: 793-802

PACE Manual Writing Group (2012) The PACE Clinic Manual: A Treatment Approach for Young People at Ultra High Risk of Psychosis. Orygen Youth Health.

Parnas J, Handest P, Jansson L, et al (2005) Anomalous subjective experience among first-admitted schizophrenia spectrum patients: empirical investigation. Psychopathology, 38: 259-67.

Perkins DO, Jeffries CD, Addington J, et al (2015) Towards a psychosis risk blood diagnostic for persons experiencing high-risk symptoms: preliminary results from the NAPLS project. Schizophrenia Bulletin, 41: $419-28$.

Phillips LJ, McGorry PD, Yuen HP, et al (2007) Medium term follow-up of a randomized controlled trial of interventions for young people at ultra high risk of psychosis. Schizophrenia Research, 96: 25-33.

Preti A, Cella M (2010) Randomized-controlled trials in people at ultra high risk of psychosis: A review of treatment effectiveness. Schizophrenia Research, 123: 30-6.

Ruhrmann S, Bechdolf A, Kuhn KU, et al (2007) Acute effects of treatment for prodromal symptoms for people putatively in a late initial prodromal state of psychosis. British Journal of Psychiatry, 191 (suppl 51): s88-95.

Ruhrmann S, Schultze-Lutter F, Salokangas RK, et al (2010) Prediction of psychosis in adolescents and young adults at high risk: results from the prospective European Prediction of Psychosis Study. Archives of General Psychiatry, 67: 241-51.

Schultze-Lutter F, Addington J, Ruhrmann S, et al (2007) Schizophrenia Proneness Instrument, Adult Version (SPI-A). Giovanni Fioriti Editore.

Stafford MR, Jackson H, Mayo-Wilson E, et al (2013) Early interventions to prevent psychosis: systematic review and meta-analysis. BMJ, 346: f185.

Sullivan HS (1927) The onset of schizophrenia. American Journal of Psychiatry, 6: 105-34.

Thompson A, Nelson B, Yung A (2011) Predictive validity of clinical variables in the 'at risk' for psychosis population: international comparison with results from the North American Prodrome Longitudinal Study. Schizophrenia Research, 126: 51-7.

Valmaggia LR, McCrone P, Knapp M, et al (2009) Economic impact of early intervention in people at high risk of psychosis. Psychological Medicine, 39: $1617-26$

van der Gaag M, Nieman DH, Rietdijk J, et al (2012) Cognitive behavioral therapy for subjects at ultra high risk for developing psychosis: a randomized controlled clinical trial. Schizophrenia Bulletin, 38: 1180-8.

van der Gaag M, Smit F, Bechdolf A, et al (2013) Preventing a first episode of psychosis: meta-analysis of randomized controlled prevention trials of 12 month and longer-term follow-ups. Schizophrenia Research, 149: $56-62$.

Yang LH, Anglin DM, Wonpat-Borja AJ, et al (2013) Public stigma associated with psychosis risk syndrome in a college population: implications for peer intervention. Psychiatric Services, 64: 284-8.

Yung AR, McGorry PD (1996) The initial prodrome in psychosis: descriptive and qualitative aspects. Australian and New Zealand Journal of Psychiatry, 30: 587-99.

Yung AR, McGorry PD (2007) Prediction of psychosis: setting the stage. British Journal of Psychiatry, 191 (suppl 51): s1-8.

Yung AR, Phillips LJ, Yuen HP, et al (2003) Psychosis prediction: 12-month follow up of a high-risk ('prodromal') group. Schizophrenia Research, 60: 21-32.

Yung AR, Phillips LJ, McGorry PD (2004a) Treating Schizophrenia in the Prodromal Phase. Taylor \& Francis.

Yung AR, Phillips LJ, Yuen HP, et al (2004b) Risk factors for psychosis in an ultra high-risk group: psychopathology and clinical features. Schizophrenia Research, 67: 131-42.

Yung AR, Yuen HP, McGorry PD, et al (2005) Mapping the onset of psychosis: the Comprehensive Assessment of At-Risk Mental States. Australian and New Zealand Journal of Psychiatry, 39: 964-71.

Yung AR, Yuen HP, Berger G, et al (2007) Declining transition rate in ultra high risk (prodromal) services: dilution or reduction of risk? Schizophrenia Bulletin, 33: 673-81.

Yung AR, Nelson B, Thompson AD, et al (2010) Should a 'risk syndrome for psychosis' be included in the DSMV? Schizophrenia Research, 120: $7-15$
MCOs

Select the single best option for each question stem

1 The ultra-high-risk (UHR) criteria:

a are based solely on clinical symptoms

b are based on disturbances in the sense of self

c include three groups that are based on clinical factors, genetic risk and functional decline

$\mathrm{d}$ are synonymous with the concept of schizotypy

e are an attempt to identify the long-term risk of psychosis.
2 A meta-analysis suggests that the rate of development of psychosis over the next 12 months in those meeting at-risk mental state criteria at baseline is around:
a $5 \%$
b $10 \%$
c $20 \%$
d $40 \%$
e $60 \%$.

3 Ethical considerations on diagnosing and treating an at-risk mental state include:

a the potential overuse of medication in 'false positives'

b stigmatising individuals

c treatments increasing the risk of development of psychosis

d pathologising potentially normal experiences

e all of the above.

4 In randomised controlled trials, the following treatment has been shown to be effective in reducing transition to psychosis:

a cognitive-behavioural therapy

b $\mathrm{N}$-acetylcysteine

c glycine

d selective serotonin reuptake inhibitors

e lithium.
5 Current guidance on treatment of someone with an at-risk mental state includes:

a treating comorbidities such as depression, if present

b formulation-based cognitive-behavioural therapy if available

c the avoidance of antipsychotic medication in the first instance unless rapid worsening of psychotic symptoms occurs, together with significant deterioration in functioning related to these symptoms and elevated risk to self or others

d practical or needs-based interventions and interventions for substance use

e all of the above. 